\title{
Airway impedance entropy and exacerbations in severe asthma
}

\author{
Sherif Gonem*, , Imraan Umar*, Daniel Burke ${ }^{\#, ~ D h a n a n j a y ~ D e s a i *, ~}$ \\ Steven Corkill*, John Owers-Bradley\#, Christopher E. Brightling* \\ and Salman Siddiqui*
}

ABSTRACT: Variability of peak flow measurements has been related to clinical outcomes in asthma. We hypothesised that the entropy, or information content, of airway impedance over short time scales may predict asthma exacerbation frequency.

66 patients with severe asthma and 30 healthy control subjects underwent impulse oscillometry at baseline and following bronchodilator administration. On each occasion, airway impedance parameters were measured at 0.2-s intervals for $150 \mathrm{~s}$, yielding a time series that was then subjected to sample entropy (SampEn) analysis.

Airway impedance and SampEn of impedance were increased in asthmatic patients compared with healthy controls. In a logistic regression model, SampEn of the resistance at $\mathbf{5 ~} \mathbf{H z}$ minus the resistance at $20 \mathrm{~Hz}$, a marker of the fluctuation of the heterogeneity of airway constriction over time, was the variable most strongly associated with the frequent exacerbation phenotype (OR 3.23 for every 0.1 increase in SampEn).

Increased airway impedance and SampEn of impedance are associated with the frequent exacerbation phenotype. Prospective studies are required to assess their predictive value.

\section{KEYWORDS: Airflow obstruction, asthma, entropy, oscillometry}

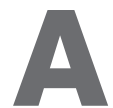
cute exacerbations of asthma account for much of the morbidity and mortality associated with this condition [1]. However, there is no currently available biomarker that can accurately predict the risk of future exacerbations. Previous studies have suggested that a geometrically self-similar airway tree may confer increased risk of asthma exacerbations and that fatal asthma is associated with a reduction in the structural complexity of the airway tree [2]. Similarly, the ventilation heterogeneity observed in asthma follows power law behaviour, which predicts catastrophic closure of small airways [3]. Therefore, characterising structural complexity may have utility in predicting asthma exacerbations.

It has been speculated that the temporal variability in lung function may also exhibit self-similarity at multiple time scales [4]. This would suggest that monitoring lung function over short time scales may provide insights into lung function variability over longer time scales of weeks to months, thus providing a more practical predictive tool for exacerbations. A number of tools have been utilised to characterise time series properties of physiological signals, including those that predict scaling and power law behaviour of information over multiple time scales and those that predict the probability of information repeating itself within a time series $[5,6]$. Fluctuations and power law behaviour observed in a time series of lung function measurements such as peak expiratory flow (PEF) may predict poor asthma control or exacerbations [7, 8]. THAMRIN et al [9] found that the degree of long-range correlation (self-similarity at different temporal length scales) in PEF measurements appeared to provide additional predictive information with respect to exacerbations in mildto-moderate asthma, but less so in severe asthma.

The forced oscillation technique (FOT) [10] provides an ideal tool for measuring airway function over time, as it allows the respiratory system to be interrogated at a high temporal resolution by delivering forced oscillations to the airways and measuring the impedance (incorporating resistance and reactance components) of the respiratory system. Dynamics of FOT time series over very short time scales (e.g. minutes) may provide additional information that predicts the behaviour of the airways over longer time scales. For instance, QuE et al. [11] plotted frequency distributions of the
AFFILIATIONS

*Institute for Lung Health, Dept of Infection, Immunity and Inflammation, University of Leicester Leicester, and

\# School of Physics and Astronomy, University of Nottingham,

Nottingham, UK.

"These authors contributed equally.

CORRESPONDENCE

S. Gonem

Respiratory Medicine

Glenfield Hospital

Leicester

LE3 9QP

UK

E-mail: sg330@le.ac.uk

Received:

Dec 302011

Accepted after revision:

Feb 132012

First published online:

March 092012 
natural logarithm of respiratory system impedance (lnZrs), measured six times per second over a 15-min period, and found that both the mean and standard deviation of the $\ln Z$ rs were higher in patients with asthma than in healthy controls. Furthermore, in healthy controls, unloading of the airway smooth muscle (ASM) induced by adoption of a supine posture in conjunction with increased ASM activation induced by methacholine (MCh) challenge led to a significant increase in the standard deviation of $\ln Z \mathrm{rs}$, recapitulating the fluctuating behaviour observed in the asthmatic airway [11]. The authors concluded that asthma may be associated with not only generalised airway narrowing but also an increased appearance of statistically unlikely airway configurations.

Entropy, a measure of increased irregularity and statistically unlikely configurations, has been utilised to characterise a variety of physiological signals [12]. Entropy measurements give a statistical probability that a series of points within a physiological signal will repeat themselves at a subsequent timepoint, within a given tolerance [13]. The entropy of FOT time series was recently investigated by VEIGA et al. [14], who found that the entropy of airflow time series was reduced in patients with asthma compared with healthy controls, and that reduced entropy was associated with increased severity of airflow obstruction. However, the possible associations between the entropy of airway impedance measurements and other clinically important patient-centred outcomes in asthma, including asthma control and exacerbations, have not been evaluated.

We hypothesised that: 1) severe asthma is characterised by altered entropy of airway resistance and reactance; and 2) entropy of impedance time series is related to exacerbation frequency in severe asthma.

\section{METHODS}

\section{Subjects}

66 patients with severe asthma fulfilling the American Thoracic Society (ATS) criteria for asthma, and with no other respiratory disease, were recruited from Glenfield Hospital (Leicester, UK) outpatients. 33 patients were at Global Initiative for Asthma (GINA) treatment step 4 and 33 at GINA treatment step 5, as previously defined [15]. 30 healthy control subjects with similar demographics were recruited from hospital staff and by local advertising. Healthy subjects had no history of respiratory disease, and had normal spirometry and MCh responsiveness. All subjects were over the age of 18 yrs. The Leicestershire and Rutland ethics committee (Leicester) approved the study and all subjects gave written informed consent.

\section{Subject characterisation}

Patients with asthma completed the Asthma Control Questionnaire (ACQ) and Asthma Quality of Life Questionnaire (AQLQ). The number of severe asthma exacerbations suffered in the previous year was recorded. Severe exacerbations were defined as an acute worsening of asthma symptoms requiring treatment with high-dose systemic corticosteroids for $\geqslant 3$ days [16]. Spirometry was performed according to ATS/European Respiratory Society (ERS) guidelines [17]. In particular, long-acting bronchodilators were withheld for $12 \mathrm{~h}$ prior to testing and short-acting bronchodilators for $4 \mathrm{~h}$. Sputum induction and cell counting were performed as previously described [18]. Exhaled nitric oxide fraction at an expiratory flow rate of $50 \mathrm{~mL} \cdot \mathrm{s}^{-1}\left(\mathrm{FeNO}_{50}\right)$ was measured using a chemiluminescence analyser (NIOX; Aerocrine, Stockholm, Sweden), according to ATS/ERS guidelines [19].

\section{Measurement of respiratory impedance}

Impedance testing was undertaken using a Jaeger MasterScreen Impulse Oscillometry (IOS) system (Viasys Healthcare $\mathrm{GmbH}$, Hoechberg, Germany), according to standard guidelines [20]. IOS was performed in the $5-35-\mathrm{Hz}$ frequency range, with impulses triggered every $0.2 \mathrm{~s}$ for $150 \mathrm{~s}$. At each time point, the resistance $(R)$ and reactance $(X)$ components of impedance were recorded at multiple frequencies from 5 to $35 \mathrm{~Hz}$, yielding time series of each variable containing $\sim 750$ data points. IOS was performed both before and after the administration of inhaled salbutamol $(400 \mu \mathrm{g})$, delivered via a metered-dose inhaler and spacer.

A variety of measures of airway calibre were derived from the mean values of the 750 data points captured (table 1): 1) $R$ at $20 \mathrm{~Hz}(R 20)$, which we interpret as a measure of the mean level of airway constriction within the bronchial tree; 2) $R$ at $5 \mathrm{~Hz}$ (R5) minus $R 20(R 5-R 20)$, which we interpret as a measure of the heterogeneity of airway narrowing throughout the bronchial tree [21]; and 3) the area under the curve of the reactance spectrum between $5 \mathrm{~Hz}$ and resonant frequency (reactance area; $A X)$, which we interpret as a measure of the heterogeneity of airway closure throughout the bronchial tree [22].

Examples of time series of $R 20$ and $R 5-R 20$ at baseline are shown in figure 1 , in a patient with severe asthma and a healthy control subject.

\section{Sample entropy analysis}

We used the sample entropy (SampEn) algorithm to evaluate complexity in the respiratory impedance time series, using a custom program downloaded from the PhysioNet online resource [23]. This measure relies on the identification of recurrent patterns within a nonstationary dynamic time series, as described in detail in the online supplementary material. Within a highly regular system, sequence matches are of greater frequency, implying lower entropy and less complexity. SampEn has emerged as a less biased metric of variability than the alternative measure approximate entropy, and is relatively independent of record length, since it does not incorporate

\section{TABLE 1 Airway impedance biomarkers}

\begin{tabular}{|c|c|}
\hline Airway impedance biomarker & Clinical interpretation \\
\hline $\mathbf{R} 20$ & Mean level of airway constriction \\
\hline$R 5-R 20$ & Heterogeneity of airway constriction \\
\hline$A x$ & Heterogeneity of airway closure \\
\hline SampEn $R_{20}$ & $\begin{array}{c}\text { Fluctuation of the mean level of } \\
\text { airway constriction over time }\end{array}$ \\
\hline SampEn $\boldsymbol{R}_{\mathbf{5}}-\boldsymbol{R} \mathbf{2 0}$ & $\begin{array}{c}\text { Fluctuation of the heterogeneity of } \\
\text { airway constriction over time }\end{array}$ \\
\hline SampEn $A x$ & $\begin{array}{c}\text { Fluctuation of the heterogeneity of } \\
\text { airway closure over time }\end{array}$ \\
\hline
\end{tabular}



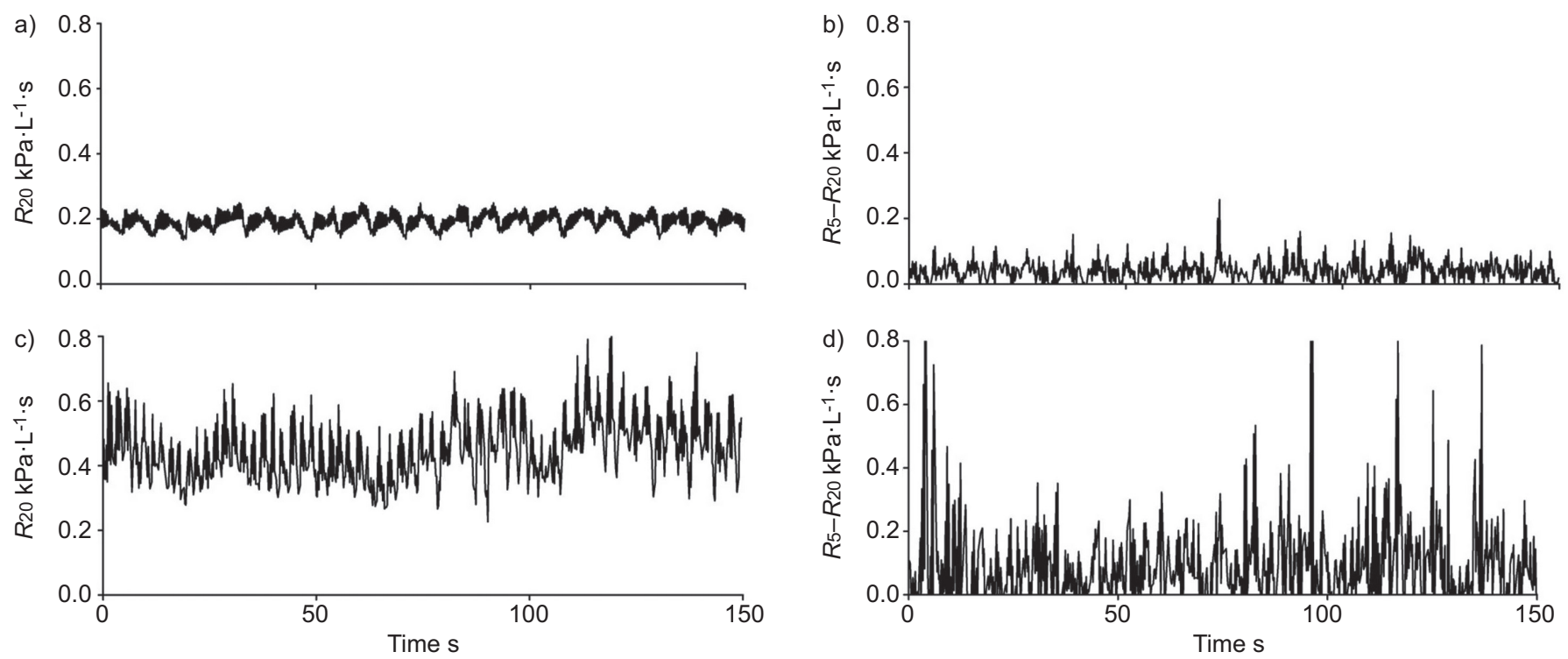

FIGURE 1. Examples of airway impedance time series. a) Resistance at $20 \mathrm{~Hz}(R 20)$ in a healthy control subject; b) resistance at $5 \mathrm{~Hz}$ minus $R 20(R 5-R 20)$ in a healthy control subject; c) $R 20$ in a patient with severe asthma; d) $R 5-R 20$ in a patient with severe asthma.

self-matches within the time series into the calculation of conditional probability [13]. We interpret SampEn of the core airway impedance markers $(R 20, R 5-R 20$ and $A X)$ to be a measure of fluctuation as a function of time (table 1). A higher value for SampEn equates to greater levels of temporal fluctuation and vice versa. Further details may be found within the online supplementary material.

\section{Statistical analysis}

Statistical analysis was performed using Prism version 5 (GraphPad, San Diego, CA, USA) and SPSS version 16.0 (SPSS Inc., Chicago, IL, USA). Colour maps were produced using Matlab R2007b (Mathworks Inc., Boston, MA, USA). Parametric data are presented as mean \pm SEM, data that were log-normally distributed were log transformed and are presented as geometric mean $(95 \% \mathrm{CI})$, and nonparametric data are presented as median (interquartile range). Unmatched groups were compared using one-way ANOVA with Bonferroni's correction or unpaired ttests for normally distributed data, Chi-squared test or Fisher's exact test for ratios, unpaired t-tests of log-transformed data for log-normally distributed data, and the Kruskal-Wallis test with Dunn's correction or the Mann-Whitney U-test for nonparametric data. Matched groups were compared using Friedman's test with Dunn's correction. A p-value of $<0.05$ was taken as the threshold for statistical significance. Spearman's correlation coefficient was used to determine the degree of correlation between impedance parameters, and principal component analysis with varimax rotation and Kaiser normalisation was used as a data reduction tool. Logistic regression analysis was performed using block entry with all selected independent variables entered at the first step.

\section{RESULTS}

\section{Subject demographics}

The clinical and demographic characteristics of the study population are shown in table 2 . The groups were well-matched for sex but differed significantly with respect to age and body mass index (BMI). However, there were no significant differences between the two asthmatic GINA 4 and GINA 5 cohorts.

\section{Severe asthma is characterised by increased heterogeneous airway constriction and closure}

Median values of heterogeneous airway constriction $(R 5-R 20)$ and closure $(A X)$ at baseline were significantly raised in both asthma groups compared with healthy controls, as shown in table 3 (R5-R20: healthy controls $0.035 \mathrm{kPa} \cdot \mathrm{L}^{-1} \cdot \mathrm{s}$, GINA 4 asthma $0.08 \mathrm{kPa} \cdot \mathrm{L}^{-1} \cdot \mathrm{s}(\mathrm{p}<0.01)$, GINA 5 asthma $0.14 \mathrm{kPa} \cdot \mathrm{L}^{-1} \cdot \mathrm{s}(\mathrm{p}<0.001)$; $A X$ : healthy controls $0.33 \mathrm{kPa} \cdot \mathrm{L}^{-1}$, GINA 4 asthma $0.935 \mathrm{kPa} \cdot \mathrm{L}^{-1}$ $(\mathrm{p}<0.05)$, GINA 5 asthma $\left.1.8 \mathrm{kPa} \cdot \mathrm{L}^{-1}(\mathrm{p}<0.001)\right)$. The mean level of airway constriction (R20) was also raised in the asthma groups compared with controls, but this effect was much less pronounced and only reached statistical significance for healthy controls versus GINA 5 asthma patients. The GINA 4 and 5 asthma groups did not differ significantly with respect to any parameter.

\section{Severe asthma is characterised by increased fluctuation (entropy) of airway impedance}

Results relating to the entropy of impedance measurements closely mirrored those of the impedance measurements themselves, as shown in table 4 . There was a progressive increase in the median baseline values of each parameter moving from the control to the GINA 4 asthma to the GINA 5 asthma groups. For instance, median SampEn of $A X$ at baseline was 0.42 in controls, 1.05 in GINA 4 asthma and 1.19 in GINA 5 asthma (p<0.0001). These observations suggest that the heterogeneity of both airway constriction and closure persists over short time scales in patients with asthma. In contrast, the mean level of airway narrowing did not fluctuate significantly in patients with asthma when compared with healthy controls.

\section{Airway impedance and entropy measures are associated} with frequent exacerbations in severe asthma

The patients with asthma were divided into those who had infrequent exacerbations, defined as fewer than two exacerbations 
TABLE 2 Clinical and demographic characteristics

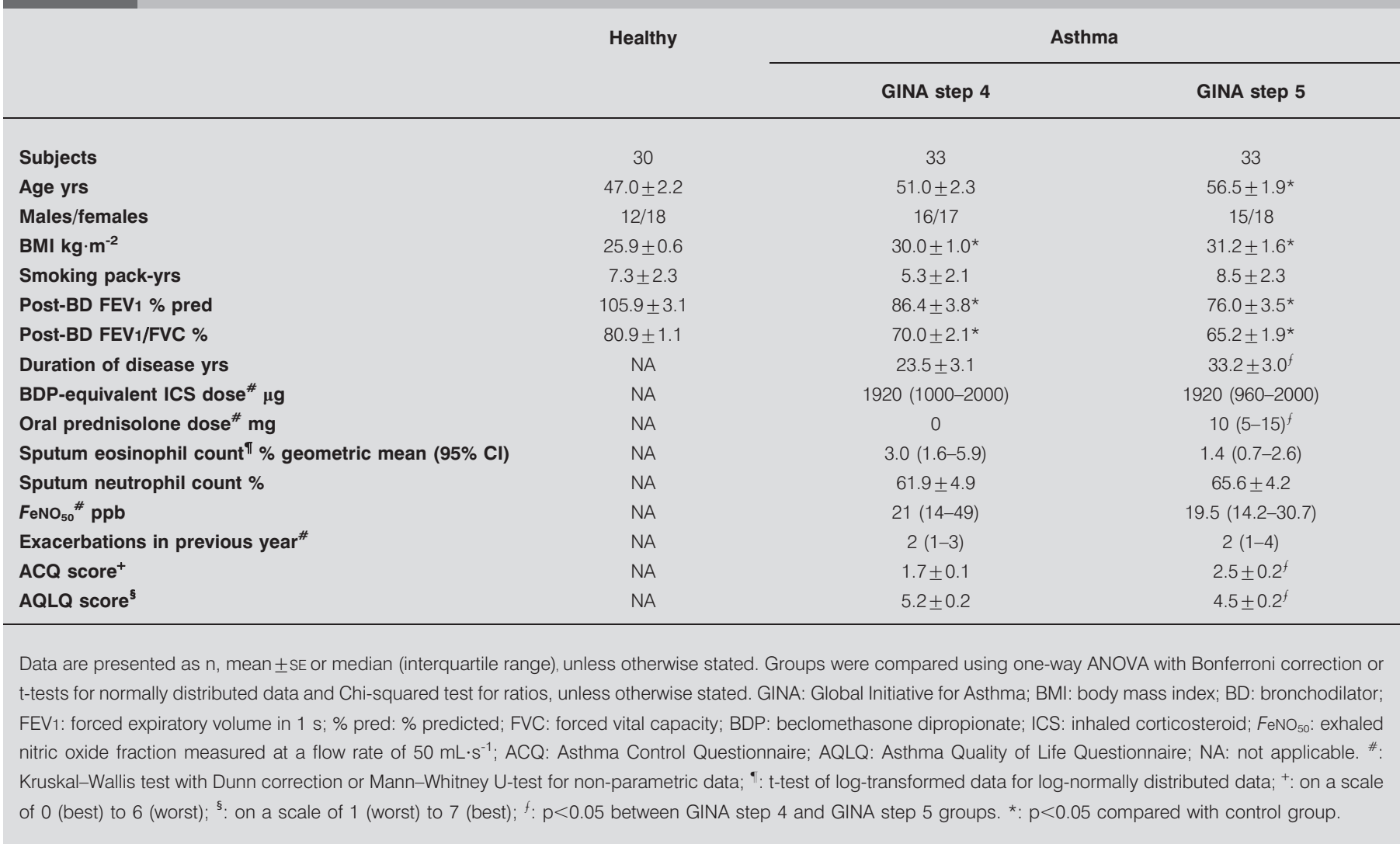

during the previous year $(n=25)$, and those who had frequent exacerbations, defined as two or more exacerbations during the previous year $(n=41)$, as shown in table 5 . The two groups did not differ significantly with respect to age, GINA category, smoking history, duration of disease, post-bronchodilator forced expiratory volume in $1 \mathrm{~s}$ (FEV1) \% predicted or FEV1/ forced vital capacity ratio, sputum eosinophil or neutrophil counts, $\mathrm{FeNO}_{50}$ or AQLQ score. However, frequent exacerbators were significantly more likely to be female than infrequent exacerbators, had a significantly increased mean BMI and had a higher mean ACQ score.

All six impedance and impedance entropy parameters were significantly raised at baseline in frequent exacerbators compared with infrequent exacerbators, as shown in table 6. For example, median $R 5-R 20$ was $0.07 \mathrm{kPa} \cdot \mathrm{L}^{-1} \cdot \mathrm{s}$ in infrequent

TABLE 3 Impedance measurements in healthy subjects and patients with asthma

\begin{tabular}{|c|c|c|c|}
\hline & $R 5-R 20 \mathrm{kPa} \cdot \mathrm{L}^{-1} \cdot \mathrm{s}$ & $A x \mathrm{kPa} \cdot \mathrm{L}^{-1}$ & $R 20 \mathrm{kPa} \cdot \mathrm{L}^{-1} \cdot \mathrm{s}$ \\
\hline \multicolumn{4}{|c|}{ Healthy controls } \\
\hline Post-BD & $0.040(0.020-0.050)$ & $0.290(0.175-0.390)^{\# \# \#}$ & $0.285(0.250-0.340)$ \\
\hline \multicolumn{4}{|l|}{ Asthma } \\
\hline \multicolumn{4}{|l|}{ GINA step 4} \\
\hline \multicolumn{4}{|l|}{ GINA step 5} \\
\hline Baseline & $0.140(0.095-0.305)^{\# \# \#}$ & $1.800(0.613-3.640)^{* \star *}$ & $0.390(0.340-0.475)^{* * *}$ \\
\hline Post-BD & $0.130(0.070-0.210)^{\# \#}$ & $1.305(0.643-2.620)^{\# \#}$ & $0.40(0.320-0.485)$ \\
\hline
\end{tabular}


TABLE 4 Sample entropy (SampEn) of impedance measurements in healthy subjects and patients with asthma

\begin{tabular}{|c|c|c|c|}
\hline & SampEn $R 5-R 20$ & SampEn $A x$ & SampEn $R 20$ \\
\hline \multicolumn{4}{|c|}{ Healthy controls } \\
\hline Post-BD & $0.004(0.001-0.021)$ & $0.39(0.19-0.59)$ & $0.021(0.007-0.060)$ \\
\hline \multicolumn{4}{|l|}{ Asthma } \\
\hline \multicolumn{4}{|l|}{ GINA step 4} \\
\hline \multicolumn{4}{|l|}{ GINA step 5} \\
\hline Baseline & $0.150(0.055-0.300)^{\# \# \#}$ & $1.19(0.80-1.45)^{\# \# \#}$ & $0.129(0.053-0.291)^{\# \# \#}$ \\
\hline Post-BD & $0.094(0.017-0.190)^{\mu}$ & $1.09(0.69-1.37)^{\bullet}$ & $0.096(0.036-0.283)$ \\
\hline
\end{tabular}

Data are presented as median (interquartile range). $R 5-R 20$ : resistance at $5 \mathrm{~Hz}$ minus resistance at $20 \mathrm{~Hz}$; $A x$ : reactance area; $R 20$ : resistance at $20 \mathrm{~Hz}$; $\mathrm{BD}$ bronchodilator; GINA: Global Initiative for Asthma. ${ }^{* *}$ : $\mathrm{p}<0.01$ between GINA step 4 and GINA step 5 groups (using Kruskal-Wallis test with Dunn's correction); ${ }^{\# \#: ~}$ $\mathrm{p}<0.01$ compared with control group (using Kruskal-Wallis test with Dunn's correction); ${ }^{\# \# \#: ~} \mathrm{p}<0.001$ compared with control group (using Kruskal-Wallis test with Dunn's correction); $\because p<0.05$ compared with baseline (using Friedman's test with Dunn's correction); ${ }^{\text {" }}: p<0.01$ compared with baseline (using Friedman's test with Dunn's correction).

and $0.13 \mathrm{kPa} \cdot \mathrm{L}^{-1} \cdot \mathrm{s}$ in frequent exacerbators $(\mathrm{p}=0.0065)$, while median SampEn R5-R20 was 0.014 in infrequent and 0.114 in frequent exacerbators $(p=0.0004)$. However, these differences were much less pronounced following bronchodilator administration (table S1).

\section{SampEn R5-R20 is independently associated with frequent exacerbations in severe asthma}

Close correlations were found between impedance and impedance entropy measurements, with $r>0.5$ and $\mathrm{p}<0.01$ for every combination of parameters, as shown in table S2 and figure S4. Principal component analysis of all the continuous variables that were associated with frequent exacerbations showed that most impedance and impedance entropy measurements loaded onto the same factor, as shown in table S3.

A logistic regression model was constructed with the presence or absence of frequent exacerbations, defined as two or more exacerbations within the previous year, as the dependent variable. Sex, BMI and ACQ score were entered into the model as independent variables, as well as one of the impedance or impedance entropy parameters. The most favourable model, chosen on the basis of the greatest predictive value in this

TABLE 5 Clinical features of asthma patients who had infrequent and frequent exacerbations

Patients with infrequent ${ }^{\#}$ exacerbations

Patients with frequent ${ }^{\text {T }}$ exacerbations

25
$51.7 \pm 2.8$
$16 / 9$
$28.1 \pm 1.3$
$16 / 9$
$6.2 \pm 2.7$
$85.8 \pm 4.4$
$67.0 \pm 2.5$
$26.8 \pm 4.2$
$2.5(1.1-5.4)$
$67.9 \pm 4.8$
$20.5(13.7-44.5)$
$1.7(0.2)$
$5.2(0.2)$

41
$55.0 \pm 1.7$
$15 / 26$
$32.1 \pm 1.2$
$17 / 24$
$7.4 \pm 1.9$
$78.2 \pm 3.3$
$67.9 \pm 1.7$
$29.3 \pm 2.6$
$1.8(1.0-3.2)$
$61.2 \pm 4.2$
$20.9(14.4-33.2)$
$2.3(0.2)$
$4.6(0.2)$

Data are presented as $\mathrm{n}$ or mean \pm SE, unless otherwise stated. Groups compared using unpaired t-tests for normally distributed data and Fisher's exact test for ratios BMI: body mass index; GINA: Global Initiative for Asthma; BD: bronchodilator; FEV1: forced expiratory volume in $1 \mathrm{~s}$; \% pred: \% predicted; FVC: forced vital capacity; $\mathrm{FeNO}_{50}$ : exhaled nitric oxide fraction measured at a flow rate of $50 \mathrm{~mL} \cdot \mathrm{s}^{-1}$; ACQ: Asthma Control Questionnaire; AQLQ: Asthma Quality of Life Questionnaire. ${ }^{\#}$ : fewer than two exacerbations during the previous year; ${ }^{\bullet}$ : two or more exacerbations during the previous year; ${ }^{+}$: unpaired t-test of log-transformed data for log-normally distributed

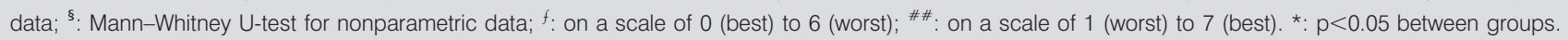


TABLE 6 Baseline impedance measurements of asthma patients who had infrequent and frequent exacerbations

\begin{tabular}{|c|c|c|c|}
\hline Impedance parameter & Patients with infrequent* exacerbations & Patients with frequent ${ }^{\text {T }}$ exacerbations & p-value ${ }^{+}$ \\
\hline Subjects n & 25 & 41 & \\
\hline SampEn $R 5-R 20$ & $0.014(0.003-0.101)$ & $0.114(0.057-0.281)$ & 0.0004 \\
\hline$A x \mathrm{kPa} \cdot \mathrm{L}^{-1}$ & $0.700(0.345-1.345)$ & $1.270(0.860-3.100)$ & 0.0041 \\
\hline SampEn $A x$ & $0.922(0.417-1.212)$ & $1.216(0.992-1.454)$ & 0.0041 \\
\hline
\end{tabular}

Data are presented as median (interquartile range), unless otherwise stated. $R 5-R 20$ : resistance at $5 \mathrm{~Hz}$ minus resistance at $20 \mathrm{~Hz}$; SampEn: sample entropy; Ax reactance area; R20: resistance at $20 \mathrm{~Hz}$. ${ }^{\#}$ : fewer than two exacerbations during the previous year; " Whitney U-test.

dataset (table S4), was that incorporating SampEn R5-R20. The parameters of this model, which correctly classified $74.2 \%$ of asthma patients as having frequent or infrequent exacerbations, are shown in table 7. SampEn $R 5-R 20$ was the only variable significantly associated with frequent exacerbations in this model $(\mathrm{p}=0.016)$, with an odds ratio of 3.23 for every 0.1 increase in SampEn R5-R20.

\section{DISCUSSION}

We have shown for the first time that increased heterogeneity of airway constriction and closure $(R 5-R 20$ and $A X)$, and increased fluctuation of these biomarkers over time (SampEn) are associated with an exacerbation-prone phenotype in patients with severe asthma. This association appears to be strongest with SampEn R5-R20, a putative marker of time-varying fluctuation of heterogeneous airway constriction. We have established that impedance time series entropy measurements are closely correlated with raw impedance values, suggesting that the raw values alone may provide useful prognostic information, but that time series analysis could be of additional value. Our data

\begin{tabular}{lcc} 
TABLE 7 & \multicolumn{2}{c}{$\begin{array}{l}\text { Logistic regression analysis of predictors of the } \\
\text { exacerbation-prone phenotype in severe asthma }\end{array}$} \\
$\begin{array}{l}\text { Predictor variable } \\
\text { OR for frequent exacerbations } \\
(\mathbf{9 5 \%} \mathbf{C l})\end{array}$ & p-value \\
\hline ACQ score & $1.227(0.628-2.4)$ & 0.549 \\
Sex & $2.618(0.781-8.783)$ & 0.119 \\
BMI $\mathbf{~ k g} \cdot \mathbf{m}^{-\mathbf{2}}$ & $1.027(0.939-1.123)$ & 0.562 \\
SampEn $\mathbf{R 5}-\mathbf{R} \mathbf{2 0}$ & $3.23(1.242-8.4)$ & 0.016 \\
\hline
\end{tabular}

Logistic regression: having frequent exacerbations (two or more in the previous year) was compared with the baseline category of having infrequent exacerbations (fewer than two in the previous year). Odds ratios are for a one-point increase in Asthma Control Questionnaire (ACQ) score, for female sex compared with the baseline category of male sex, for a $1-\mathrm{kg} \cdot \mathrm{m}^{-2}$ increase in body mass index (BMI) and for a 0.1 increase in sample entropy (SampEn) of resistance at $5 \mathrm{~Hz}(R 5)$ minus resistance at $20 \mathrm{~Hz}(R 20)$. " : on a scale of 0 (best) to 6 (worst). suggest that airway closure and narrowing (in particular, heterogeneous time fluctuation behaviour) identifies patients at the greatest risk of exacerbations. Indeed, a 0.1 increase in sample entropy of heterogeneous airway constriction (R5-R20) was associated with a 3.2-fold increase in the risk of an exacerbation. It has previously been shown that minimal heterogeneity of airway constriction can result in catastrophic shifts in ventilation to particular lung regions [24]. We speculate that the fluctuations in $R$ and $X$ we observed in patients with severe asthma represent such step-wise shifts in airway patency, occurring over a time scale of seconds, and that patients who exhibit such fluctuations may be constantly on the cusp of asthma exacerbations. In such patients, small perturbations in smooth muscle tone, for instance caused by minor allergen exposure or a viral upper respiratory tract infection, may result in life-threatening airway constriction and closure.

\section{Significance of the impedance parameters R20, R5-R20 and $\mathrm{Ax}$}

It has previously been shown that in severe asthma, the general level of airway resistance is raised, and that there is an additional increase in $R$ at low oscillation frequencies [25]. This frequency dependence of $R$ is thought to be due to heterogeneous airway constriction, since mathematical modelling indicates that even severe homogeneous constriction would produce an elevated baseline $R$ but not frequency dependence [25]. Furthermore, an image-functional modelling approach has suggested that the ventilation defects and frequency-dependence of $R$ and elastance seen in asthma can only be explained by small airway constriction, or a combination of large and small airway constriction [26]. In this study, we used $R 20$ to represent general airway resistance and $R 5-R 20$ to represent frequency-dependence of resistance, a marker of heterogeneous airway constriction. $A x$ is thought to be a marker of airway closure, since such closure results in peripheral airway capacitive properties not being measured, thus increasing the effective elastance of the respiratory system [22].

\section{Impedance and impedance entropy parameters in healthy subjects and patients with asthma}

In line with previous studies [27, 28], we found significant increases in $R_{5}-R 20$ and $A X$, and to a lesser extent $R 20$, in patients with severe asthma compared with healthy controls, 
suggesting that the baseline level of airway narrowing, and the accompanying heterogeneity of narrowing and closure, are increased in patients with severe asthma. We were unable to demonstrate a difference in these parameters between patients with severe asthma established on maintenance oral corticosteroids (GINA treatment step 5) and patients on high-dose inhaled corticosteroids (GINA treatment step 4), suggesting that these processes may be resistant to corticosteroid therapy.

The SampEn parameters were higher at baseline in the asthma groups than in the control group, although this only reached statistical significance for the GINA 5 versus control comparison. Close correlations were seen between impedance measurements and their respective SampEn parameters, as shown in table S2 and figure S4, suggesting that entropy of airway impedance is strongly associated with baseline airway calibre.

\section{Determinants of the exacerbation-prone asthma phenotype}

In our cohort of patients with asthma, we found that those who had suffered two or more exacerbations in the previous year were significantly more likely to be female, and had a significantly greater BMI and ACQ score. Obesity [29, 30] and reduced asthma control [29] have previously been associated with asthma exacerbations, although a recent large observational study of asthma outcomes [29] did not find female sex to be a significant predictor of having had a recent severe exacerbation. Interestingly, we found that markers of eosinophilic airway inflammation were no higher in patients with frequent compared with infrequent exacerbations, suggesting that the excess exacerbations in the former group were due to factors other than uncontrolled eosinophilic airway inflammation. Of note, all patients in this study were being treated with high-dose inhaled or long-term oral corticosteroids, which would be expected to suppress eosinophilic airway inflammation [31].

Previous studies have shown that fixed airflow obstruction is a risk factor for frequent exacerbations [32]. Although postbronchodilator FEV1 was numerically lower in our patients with frequent exacerbations than those without frequent exacerbations ( 78.2 versus $85.8 \%$ pred), this result did not reach statistical significance. In contrast, all three of the impedance parameters at baseline, namely $R 5-R 20, A X$ and $R 20$, were significantly higher in those with frequent exacerbations compared with those without. Very similar results were obtained for the SampEn of impedance parameters. However, as shown in table S1, post-bronchodilator values of the parameters were much less discriminatory, suggesting that pre-bronchodilator values may be more valuable as predictors of clinical outcome. This is concordant with the results of SHI et al. [33], who found that pre-bronchodilator impedance parameters could more effectively discriminate between children with well-controlled and poorly-controlled asthma than post-bronchodilator values.

A logistic regression model (table 6) showed that SampEn R5$R 20$ was independently associated with frequent exacerbations. However, substitution of this with any one of the other impedance or impedance entropy parameters resulted in similar model performance (table S4). This suggests that calculation of the SampEn of impedance time series may provide only a small amount of additional information over and above the mean values of the impedance parameters themselves.

\section{Limitations of the study}

We have shown that increased airway impedance parameters are associated with frequent asthma exacerbations. However, our conclusions are based on retrospective data, and thus prospective studies are required in order to validate the predictive capacity of the impedance measurements. Nevertheless, our results suggest that airway impedance measurements represent a marker of exacerbation risk that may be used in clinical practice or as an outcome measure in clinical trials.

In addition, further work is required to interpret the structural basis of the impedance parameters derived from impulse oscillometry $(R 20, R 5-R 20$ and $A x)$. Inverse modelling approaches $[21,22]$ and ex vivo airway models may provide a deeper understanding.

Finally, in this study we chose to examine the sample entropy of impedance time series as a marker of the temporal fluctuation of airway calibre. However, a number of other techniques exist to analyse time series data, including de-trended fluctuation analysis, a measure of long-range scaling [5, 6], and dynamic systems analysis [34], and it is possible that one or more of these alternative techniques may provide greater predictive power. Future studies should investigate this possibility and further refine these novel fluctuation biomarkers against clinically important patient-centred outcomes.

\section{STATEMENT OF INTEREST}

Statements of interest for S. Siddiqui and C. E. Brightling can be found at www.erj.ersjournals.com/site/misc/statements.xhtml

\section{REFERENCES}

1 Dougherty RH, Fahy JV. Acute exacerbations of asthma: epidemiology, biology and the exacerbation-prone phenotype. Clin Exp Allergy 2009; 39: 193-202.

2 Boser SR, Park H, Perry SF, et al. Fractal geometry of airway remodeling in human asthma. Am J Respir Crit Care Med 2005; 172: 817-823.

3 Mauroy B, Filoche M, Weibel ER, et al. An optimal bronchial tree may be dangerous. Nature 2004; 427: 633-636.

4 Frey U, Maksym G, Suki B. Temporal complexity in clinical manifestations of lung disease. J Appl Physiol 2011; 110: 1723-1731.

5 Perkiömäki JS, Mäkikallio TH, Huikuri HV. Fractal and complexity measures of heart rate variability. Clin Exp Hypertens 2005; 27: 149-158.

6 Seely AJ, Macklem PT. Complex systems and the technology of variability analysis. Crit Care 2004; 8: R367-R384.

7 Frey U. Predicting asthma control and exacerbations: chronic asthma as a complex dynamic model. Curr Opin Allergy Clin Immunol 2007; 7: 223-230.

8 Frey U, Brodbeck T, Majumdar A, et al. Risk of severe asthma episodes predicted from fluctuation analysis of airway function. Nature 2005; 438: 667-670.

9 Thamrin C, Nydegger R, Stern G, et al. Associations between fluctuations in lung function and asthma control in two populations with differing asthma severity. Thorax 2011; 66: 1036-1042.

10 Goldman MD, Saadeh C, Ross D. Clinical applications of forced oscillation to assess peripheral airway function. Respir Physiol Neurobiol 2005; 148: 179-194.

11 Que CL, Kenyon CM, Olivenstein R, et al. Homeokinesis and short-term variability of human airway caliber. J Appl Physiol 2001; 91: 1131-1141.

12 Thamrin C, Frey U. Complexity and respiratory growth: a developing story. J Appl Physiol 2009; 106: 753-754. 
13 Richman JS, Moorman JR. Physiological time-series analysis using approximate entropy and sample entropy. Am J Physiol Heart Circ Physiol 2000; 278: H2039-H2049.

14 Veiga J, Lopes AJ, Jansen JM, et al. Airflow pattern complexity and airway obstruction in asthma. J Appl Physiol 2011; 111: 412-419.

15 Global Initiative for Asthma. Global Strategy for Asthma Management and Prevention. www.ginasthma.org/uploads/users/files/ GINA_Report2011_May4.pdf Date last updated: May 4, 2011.

16 Reddel HK, Taylor DR, Bateman ED, et al. An official American Thoracic Society/European Respiratory Society statement: asthma control and exacerbations: standardizing endpoints for clinical asthma trials and clinical practice. Am J Respir Crit Care Med 2009; 180: 59-99.

17 Miller MR, Hankinson J, Brusasco V, et al. Standardisation of spirometry. Eur Respir J 2005; 26: 319-338.

18 Pavord ID, Pizzichini MM, Pizzichini E, et al. The use of induced sputum to investigate airway inflammation. Thorax 1997; 52: 498-501.

19 American Thoracic Society, European Respiratory Society. ATS/ ERS recommendations for standardized procedures for the online and offline measurement of exhaled lower respiratory nitric oxide and nasal nitric oxide. Am J Respir Crit Care Med 2005; 171: 912-930.

20 Oostveen E, MacLeod D, Lorino $\mathrm{H}$, et al. The forced oscillation technique in clinical practice: methodology, recommendations and future developments. Eur Respir J 2003; 22: 1026-1041.

21 Kaminsky DA, Irvin CG, Lundblad L, et al. Oscillation mechanics of the human lung periphery in asthma. J Appl Physiol 2004; 97: 1849-1858.

22 Thorpe CW, Bates JH. Effect of stochastic heterogeneity on lung impedance during acute bronchoconstriction: a model analysis. J Appl Physiol 1997; 82: 1616-1625.

23 Goldberger AL, Amaral LA, Glass L, et al. PhysioBank, PhysioToolkit, and PhysioNet: components of a new research resource for complex physiologic signals. Circulation 2000; 101: E215-E220.
24 Venegas JG, Winkler T, Musch G, et al. Self-organized patchiness in asthma as a prelude to catastrophic shifts. Nature 2005; 434: 777-782.

25 Lutchen KR, Jensen A, Atileh $\mathrm{H}$, et al. Airway constriction pattern is a central component of asthma severity: the role of deep inspirations. Am J Respir Crit Care Med 2001; 164: 207-215.

26 Tgavalekos NT, Tawhai M, Harris RS, et al. Identifying airways responsible for heterogeneous ventilation and mechanical dysfunction in asthma: an image functional modeling approach. J Appl Physiol 2005; 99: 2388-2397.

27 Kanda S, Fujimoto K, Komatsu Y, et al. Evaluation of respiratory impedance in asthma and COPD by an impulse oscillation system. Intern Med 2010; 49: 23-30.

28 Park JW, Lee YW, Jung YH, et al. Impulse oscillometry for estimation of airway obstruction and bronchodilation in adults with mild obstructive asthma. Ann Allergy Asthma Immunol 2007; 98: 546-552.

29 Miller MK, Lee JH, Miller DP, et al. Recent asthma exacerbations: a key predictor of future exacerbations. Respir Med 2007; 101: 481-489.

30 Mosen DM, Schatz M, Magid DJ, et al. The relationship between obesity and asthma severity and control in adults. J Allergy Clin Immunol 2008; 122: 507-511.

31 Berry M, Morgan A, Shaw DE, et al. Pathological features and inhaled corticosteroid response of eosinophilic and non-eosinophilic asthma. Thorax 2007; 62: 1043-1049.

32 Contoli M, Baraldo S, Marku B, et al. Fixed airflow obstruction due to asthma or chronic obstructive pulmonary disease: 5-year follow-up. J Allergy Clin Immunol 2010; 125: 830-837.

33 Shi Y, Aledia AS, Tatavoosian AV, et al. Relating small airways to asthma control by using impulse oscillometry in children. J Allergy Clin Immunol 2012; 129: 671-678.

34 Muskulus M, Slats AM, Sterk PJ, et al. Fluctuations and determinism of respiratory impedance in asthma and chronic obstructive pulmonary disease. J Appl Physiol 2010; 109: 1582-1591. 